\title{
YcjW: a transcription factor that controls the emergency generator of $\mathrm{H}_{2} \mathrm{~S}$ in $E$. coli.
}

Lyly Luhachack ${ }^{1}$, Ilya Shamovsky ${ }^{1}$, Evgeny Nudler ${ }^{1,2}$

1 Department of Biochemistry and Molecular Pharmacology, New York University School of Medicine, New York, NY, 10016, USA

2 Howard Hughes Medical Institute, New York University School of Medicine, New York, NY, 10016, USA. Evgeny.Nudler@nyumc.org.

\section{Abstract}

Hydrogen sulfide $\left(\mathrm{H}_{2} \mathrm{~S}\right)$ is a ubiquitous gaseous molecule that is endogenously produced in both eukaryotes and prokaryotes. Its role as a pleiotropic signaling molecule has been well characterized in mammals ${ }^{1,2}$. In contrast, the physiological role of $\mathrm{H}_{2} \mathrm{~S}$ in bacteria only recently became apparent; $\mathrm{H}_{2} \mathrm{~S}$ acts as a cytoprotectant against antibiotics-induced stress and affect the cell's ability to maintain redox homeostasis ${ }^{3-5}$. In $E$. coli, endogenous $\mathrm{H}_{2} \mathrm{~S}$ production is primarily dependent on 3-mercaptopyruvate sulfurtransferase (3MST), encoded by $m s t A$, previously known as $s s e A^{3,4}$. Here, we show that cells lacking $3 \mathrm{MST}$ acquired a unique phenotypic suppressor mutation resulting in compensatory $\mathrm{H}_{2} \mathrm{~S}$ production and tolerance to antibiotics and oxidative stress. Using whole genome sequencing, we mapped a non-synonymous single nucleotide polymorphism (SNP) to uncharacterized Lacl-type transcription factor, YcjW. We identified transcriptional regulatory targets of YcjW and discovered a major target, thiosulfate sulfurtransferase PspE, as an alternative mechanism for $\mathrm{H}_{2} \mathrm{~S}$ biosynthesis. Deletion of pspE was sufficient to antagonize phenotypic suppression. Our results reveal a complex interaction between cell metabolism and $\mathrm{H}_{2} \mathrm{~S}$ production and the role, a hithero uncharacterized transcription factor, YcjW, plays in linking the two. 
Main

$\mathrm{H}_{2} \mathrm{~S}$ can be generated in numerous pathways-both enzymatically and non-enzymatically and from various substrates beyond cysteine ${ }^{6}$. The main pathway by which $E$. coli generate $\mathrm{H}_{2} \mathrm{~S}$ when grown aerobically in nutrient rich LB is via 3MST, encoded by $m s t A^{3,4}$. Phenotypic consequences of decreased $\mathrm{H}_{2} \mathrm{~S}$ production include greater susceptibility to multiple classes of antibiotics and $\mathrm{H}_{2} \mathrm{O}_{2}{ }^{3,4,7}$. However, we discovered that antibiotics-sensitive strain $\Delta m s t A$ reverted to the resistant phenotype of the isogenic parent when challenged with different antibiotics. The $\Delta m s t A$ variant, referred to as $\Delta m s t A$-sup in this study, was indistinguishable from wild type in time-kill assay analysis and growth curves of cells exposed to gentamicin, nalidixic acid and carbenicillin (Fig. 1a and Supplementary Fig.1). Furthermore, this strain also had increased tolerance to hydrogen peroxide $\left(\mathrm{H}_{2} \mathrm{O}_{2}\right)$, compared to its still sensitive parent $\Delta m s t A$ strain (Fig. $1 \mathrm{~b}$ ). Using both the classic lead acetate reactivity test for $\mathrm{H}_{2} \mathrm{~S}$ detection and a fluorescentbased probe, $\mathrm{WSP}^{8}$, we confirmed that this phenotypic reversion was concurrent with increased $\mathrm{H}_{2} \mathrm{~S}$ production, comparable to wild type (Fig. 1C). In contrast, significant levels of $\mathrm{H}_{2} \mathrm{~S}$ remained undetectable in $\Delta m s t A$ till $\mathrm{OD}_{600} 1.5$.

We utilized whole genome sequencing to identify possible SNPs coding regions that could be responsible for the observed phenotypic suppression. We mapped and validated by PCR a single missense mutation unique to $\Delta m s t A$-sup to uncharacterized transcription factor, $y c j W$. The nucleotide substitution, $\mathrm{G}$ to $\mathrm{A}$ on the coding strand, results in an amino acid change from serine to asparagine at residue 258 (Fig. 2a).

YcjW is annotated as a putative member of the Lacl/GalR family of repressors that are largely responsible for carbohydrate metabolism. Common features of the family include an N-terminal helix-turn-helix DNA-binding domain, a linker domain, and a Cterminal ligand-binding domain ${ }^{9}$. To investigate SNP functionality, we constructed two strains, bearing a plasmid expressing either wild type YcjW (pLLY1) or S258N YcjW (pLLSN1), in the background of $\Delta m s t A / \Delta y c j W$. Figure $2 \mathrm{~b}$ shows that only plasmidexpressed mutated $\mathrm{YcjW}$ is able to restore $\mathrm{H}_{2} \mathrm{~S}$ production, quantitated by utilizing the WSP5 probe and qualitatively shown by lead acetate assay. Furthermore, only $\Delta m s t A / \Delta y c j W ; \mathrm{P}_{\mathrm{LL}}-y c j W(\mathrm{~S} 258 \mathrm{~N})$ have an increased survival rate when challenged with gentamicin, $\mathrm{H}_{2} \mathrm{O}_{2}$ and nalidixic acid (Fig. $2 \mathrm{c}$ and $2 \mathrm{~d}$ and Supplementary Fig. 2). Thus, we 
confirm that S258N YcjW in $\Delta m s t A$-sup is responsible for the increased hydrogen sulfide production and antibiotics and oxidative stress tolerance relative to $\Delta m s t A$.

To identify transcriptional targets of YcjW, we performed ChIP-seq using an antibody against chromosomal 3xFLAG-tagged YcjW from wild type, and $\Delta m s t A$ cells, and 3xFLAG-tagged YcjW S258N from $\Delta m s t A$-sup cells. Figure 3a shows representative peaks identified by MACS $2^{10}$ from aligned sequence reads. The most enriched regions, for all three strains, are at two sites near $y c j W$; the first site is before the translation start site of $y c j M$ but after a predicted transcription start site and the second lies between $y c j T$ and ycjU. The binding motifs for Lacl type family transcription factors are typically palindromes with a conserved central CG pair ${ }^{11}$. Recently, Zuo and Stormo experimentally tested the predicted binding motif for $Y_{c j W}{ }^{12}$. Combined with our analysis of peak summits, we found the same sequence in our data. Using the putative binding sequence, we further restricted peaks to ones containing the conserved $14 \mathrm{bp}$ motif, allowing up to three mismatches and with a fold enrichment greater than 5 . With those criteria, we identified two additional peaks specifically in $\Delta m s t A$, that are not enriched in wild type or $\Delta m s t A-s u p$. The two sites are near the promoter of narP, encoding a two-component nitrate/nitrite response system and the other is located within the coding sequence of $c y a A$, encoding adenylate cyclase (Supplementary Fig. 3).

We then validated transcription factor binding through electrophoretic mobility shift assay (EMSA). We designed 50bp DNA probes containing the predicted binding sequence in the center. The YcjW protein reduced the mobility of the upstream ycjM DNA probe at about a 1:0.5 DNA:protein ratio. Increasing amounts of protein corresponded to an increase in YcjW-DNA complex (Fig. 3b). YcjW (S258N) also reduced DNA probe mobility at the same DNA:protein ratio, using narP probe (Fig. $3 \mathrm{c}$ ). Titration of the normal protein and S258N YcjW showed that they both bound DNA probe starting at a DNA:protein ratio of 1:0.5. At a ratio of 1:2, no free DNA probe could be detected.

The region downstream of $y c j M$ contains a predicted operon consisting of 10 genes-ycjMNOPQRSTUV and ompG. To test functionality of transcription factor-DNA binding to gene expression, we determined the amount of relative mRNA fold change using qRT-PCR. In $\triangle y c j W$, representative genes, $y c j M, y c j T, y c j U$, and ompG are 
significantly upregulated, confirming that $\mathrm{YcjW}$ is a repressor (Fig. 3d). The absence of YcjW results in constitutive derepression of its regulatory transcriptional targets. The same genes exhibit a similar pattern, increased expression, in $\Delta y c j W ; P_{L L}-y c j W S 258 \mathrm{~N}$ relative to wild type MG1655 but not to the extent of its isogenic parent, $\Delta y c j W$. Consistent with $\Delta y c j W ; \mathrm{P}_{\mathrm{LL}}-y c j W \mathrm{~S} 258 \mathrm{~N}$, those genes are also upregulated in $\Delta m s t A$-sup, but not $\Delta m s t A$, suggesting that $S 258 \mathrm{~N}$ YcjW affects DNA occupancy in vivo but not necessarily in vitro. Mutational analyses of other Lacl-TFs have demonstrated that a single amino acid change in the C-terminal can alter effector or co-repressor binding and therefore DNA affinity at target sites ${ }^{13,14}$. The SNP is located in the C-terminal effector pocket of the protein, thus raising the possibility that it broadens specificity of inducer recognition, co-repressor binding affinity or oligomerimerization.

qRT-PCR of narP and cyaA showed no significant change in $\triangle y c j W$. A small subset of genes regulated by NarP was tested as downstream targets (Supplementary Fig. 4). Two genes, nrfA and ydhU did have a modest increase while two others didn't. NarP regulation however is complex and involves multiple regulators. Therefore, it is difficult to assess if $\mathrm{YcjW}$-binding upstream of narP and cyaA is functional.

Because many Lacl-type repressors act locally in response to some specific effector, we sought to identify the inducer for YcjW by considering its targets. YcjT is homologous to kojibiose phosphorylase from Thermoanerobacter brockii and Pyrococcus sp. Strain ST04 ${ }^{15,16}$. Kojibiose phosphorylase can reversibly catabolize kojibiose to D-glucose and beta-D-glucose 1 phosphate. The downstream gene, ycjU, has experimentally been shown to encode a beta-phosphoglucomutase ${ }^{17}$. Again, utilizing EMSA, we tested to see if kojibiose is the effector molecule for YcjW. The addition of kojibiose at $1 \mathrm{mM}$ disrupts the YcjW-DNA complex (Fig. 3e). Other disaccharides tested in excess of up to ten times, trehalose and sucrose, did not affect binding. However, attempts to grow E. coli K-12 MG1655 on minimal media with kojibiose as the sole carbon source were unsuccessful (data not shown and ${ }^{18}$ ). Growth on EZ Rich Defined media supplemented with kojibiose as the carbon source did grow but had a rather pronounced defect. Deletion of $y c j W$ did not improve growth rates either (Supplementary Fig. 5). However, the concentration of kojibiose added to media is limited by its low solubility. It is possible that a higher concentration of kojibiose supplied would support enhanced growth. Taken together, our results indicate that kojibiose might 
not be the natural inducer of YcjW but perhaps some derivative of kojibiose. Recently, the substrate for YcjM was identified as glucosylglycerate, alongside kojibiose for YcjT 18. Glucosylglycerate is an osmoprotectant in bacteria and archaea and accumulates under salt stress and limited nitrogen availability ${ }^{19,20}$. However, most of our experiments were conducted in LB with amino acids constituting the main carbon source. We find it unlikely that synthesis of either the glycoside or disaccharide could occur without the appropriate substrate, and therefore is not likely involved in $\Delta m s t A$ phenotypic suppression.

While it is not directly evident how a cluster of carbohydrate catabolic genes regulated by $\mathrm{YcjW}$ can lead to an alternative pathway for $\mathrm{H}_{2} \mathrm{~S}$ production, $p s p E$ encoding a thiosulfate sulfurtransferase, lies immediately upstream of ycjM. PspE has mercaptopyruvate sulfurtransferase activity, albeit low compared to thiosulfate ${ }^{21}$. It is part of a cluster of genes known as the phage shock operon, consisting of $p s p A B C D E$. Although it can be co-regulated with the other $p s p$ genes, it can also be transcribed independently from its own promoter ${ }^{22}$. PspF is the transcriptional activator and phage shock protein $A$, encoded by $p s p A$, negatively regulates $\mathrm{PspF}^{23}$.

pspE mRNA expression is significantly increased in $\Delta m s t A$-sup and $\Delta y c j W$ relative to wild type cells. In contrast, there are no significant differences in relative expression of the other two thiosulfurtransferase genes (Fig. 4a). Furthermore $\mathrm{H}_{2} \mathrm{~S}$ production is undetectable during early exponential and mid-log phase in $\Delta m s t A$ sup/ $\Delta p s p E$ (Fig. 4b). However, at late logarithmic phase, $\mathrm{H}_{2} \mathrm{~S}$ levels are now detectable to the same degree as MG1655 and $\Delta m s t A$-sup. In addition, overnight incubation with lead acetate strips shows no discernable difference in $\mathrm{H}_{2} \mathrm{~S}$ extracellular production between the three strains (Supplementary Fig. 6). We conclude from the significant delay of $\mathrm{H}_{2} \mathrm{~S}$ generation in $\Delta m s t A$-sup/ $\Delta p s p E$ that $\mathrm{PspE}$ is capable of generating $\mathrm{H}_{2} \mathrm{~S}$ in early growth phases as observed in $\Delta m s t A$-sup. However, at later growth stages, another pathway for $\mathrm{H}_{2} \mathrm{~S}$ production is activated and/or PspE is no longer sufficient. Moreover, $\Delta m s t A$-sup/ $\Delta p s p E$ also has increased sensitivity to gentamicin treatment compared to $\Delta m s t A$-sup and $E$. coli MG1655 but not quite as sensitive as $\Delta m s t A$. Overexpression of PspE in $\Delta m s t A$ increases survival rate but only to the extent of $\triangle m s t A$ sup/ $\Delta p s p E$, not $\Delta m s t A$-sup or wild type (Fig. 4c). Altogether, we conclude that the SNP in $y c j W$ resulted in increased expression of $p s p E$ in $\Delta m s t A$-sup. This is sufficient but not 
wholly responsible for increased $\mathrm{H}_{2} \mathrm{~S}$ biosynthesis and in turn, the phenotypic suppression observed in $\Delta m s t A$-sup. We propse a model wherein, E.coli cells lacking MstA acquires a SNP in transcription factor YcjW. The SNP imparts moderate constitutive expression of YcjW targets, one of which is $p s p E$. Thiosulfate sulfurtransferase PspE is then able to increase $\mathrm{H}_{2} \mathrm{~S}$ production in $\Delta m s t A$, and subsequently protect the cells from antibiotics and $\mathrm{H}_{2} \mathrm{O}_{2}$ induced stress (Fig. 4d).

The region upstream of $p s p E$ does not contain a strong binding motif for YcjW, nor do the regions flanking regulators PspA and PspF. This is not entirely unexpected since none of the other genes tested in the psp operon were upregulated in $\Delta m s t A$-sup or $\Delta y c j W$ (Supplementary Fig. 6). Additionally, the moderate but significant increase of pspE mRNA in $\triangle y c j W$, in comparison to ycjMNOPQRSTUV-ompG, suggests a more complicated interaction than direct DNA-binding. It may be indicative of "leaky" expression driven by the proximity of the 3'end of $p s p E$ to predicted transcription start sites for $y c j M$ and the binding site of YcjW. Perhaps, YcjW acts via a co-repressor. Another possibility is that the metabolic state of $\Delta m s t A$-sup with mutated $y c j W$ induces $p s p E$ as a consequence. And finally, the enzyme(s) that is induced in $\Delta m s t A$-sup; $\Delta p s p E$, after exponential growth, has not been identified and remains a target for future studies.

Altogether, our results reveal an alternative $\mathrm{H} 2 \mathrm{~S}$ generator (PspE) that is mobilized as a result of rapid evolutional adaptation to antibacterial stress. The SNP in YcjW, regulating metabolism to at least two rare sugars, presents an interesting link between sulfur metabolism and carbon availability. Moreover, a SNP in YcjW reflects the striking genetic plasticity employed by bacteria to promptly adapt to environmental changes and stimuli and highlights the survival advantage imparted by endogenous $\mathrm{H}_{2} \mathrm{~S}$.

Acknowledgments. This work was supported by the NIH grant R01 GM126891, Blavatnik Family Foundation, and the Howard Hughes Medical Institute.

\section{Methods}

\section{General growth conditions}


For the general cultivation of E.coli, strains were grown in LB broth supplemented with kanamycin (50ug ml-1), or chloramphenicol (30ug ml $\mathrm{m}^{-1}$ ) as appropriate. Growth on solid medium contained $1.5 \%$ agar added to LB. Where noted, MOPS EZ Rich Defined Medium Kit (Teknova) was used in place of $L^{24}$. Cysteine solutions were prepared immediately before use, as needed.

\section{Construction of strains and plasmids}

For a list of all strains used throughout this work, refer to Table 1.1. BW25112 and its derivatives are from the E. coli Keio Knockout Collection ${ }^{25}$ (Thermo Scientific). Introduction of new mutations into E.coli MG1655 were achieved through P1 transduction as previously described ${ }^{26}$. Temperature-sensitive FLP recombinase plasmid pCP20 was used for the excision of selective markers as needed ${ }^{27}$. All constructs were verified with PCR and sequencing. Primers used throughout this study are listed in Table 1.2.

To generate pLLY1, ycjW was PCR amplified from E.coli MG1655 using primers LL10 and LL11 and cloned into pACYC184 plasmid (NEB) using the Gibson Assembly Mastermix, according to the manufacturer's protocol (NEB). Plasmid pLLSN3 was generated as above except ycjW was PCR amplified from mstA-sup. The Q5 SiteDirected Mutagenesis Kit (NEB) was used to generate pLLSN1 from pLLY1, according to manufacturer's protocol.

Transformations were performed using the $\mathrm{CaCl}_{2}$ competent cell protocol ${ }^{28}$. All plasmids were sequenced for verification.

Addition of 3xFLAG tag to $y c j W$ at its chromosomal locus was achieved as previously described, with slight modifications ${ }^{29}$. Briefly, primers pLL14 and pLL15 were used to PCR amplify $\mathrm{Cm}^{\mathrm{R}}$ cassette from pKD4. PCR product was transformed into appropriate electrocompetent strains.

\section{$\mathrm{H}_{2} \mathrm{~S}$ detection}

End-point detection of $\mathrm{H}_{2} \mathrm{~S}$ production by lead acetate strips were performed as previously described ${ }^{3}$. Test strips were purchased from Sigma-Aldrich. Monitoring $\mathrm{H}_{2} \mathrm{~S}$ generation with the WSP5 fluorescent probe followed a modified protocol from Peng et al. ${ }^{8}$ Briefly, cells were grown in LB at $37^{\circ} \mathrm{C}$ to desired $\mathrm{OD}_{600}$ and aliquots of approximately $4 \times 10^{8}$ cells were taken. The extinction coefficient used for calculations is $\mathrm{OD}_{600}$ of 1.0 is equal to $8 \times 10^{8}$ cells. A working solution of WSP5 was made immediately before use and added to cells for a final concentration of $10 \mathrm{uM}$. Samples were incubated 
at $37^{\circ} \mathrm{C}$ for 30 minutes and then washed in PBS buffer, $\mathrm{pH} 7.4$, to remove excess probe. Cells were resuspended in PBS buffer and incubated at room temperature for 30 minutes. Cytation3 (Biotek) was used to take fluorescent readings, at excitation $500 \mathrm{~nm}$ and emission $533 \mathrm{~nm}$. All experiments were repeated for a total of three times. Background values were subtracted during analysis.

\section{Time-kill assay and growth curves}

Overnight cultures of $E$. coli were diluted 1:300 into fresh media and grown to an $O_{600}$ of $\sim 0.2$. A $1 \mathrm{ml}$ aliquot was serially diluted and plated onto LB agar plates to determine initial colony forming units per $\mathrm{ml}\left(\mathrm{cfu} \mathrm{ml}{ }^{-1}\right)$ after overnight incubation at $37^{\circ} \mathrm{C}$. Antibiotics were added to the cultures at indicated concentrations. Aliquots of $1 \mathrm{ml}$ were collected at specified time intervals, serially diluted and plated. Results from three independent experiments were plotted in GraphPad version 5.0.

Growth curves were generated from Bioscreen $\mathrm{C}$ automated growth analysis system as previously described ${ }^{3}$. Antibiotics were purchased from Sigma-Aldrich or Gold Biotechnology.

\section{Whole genome sequencing}

Overnight cultures of $E$. coli cells were used for genomic DNA isolation. The MasterPure Complete DNA Purification Kit (Epicentre) was used to purify DNA according to the manufacturer's protocol. DNA samples were quantified using the Quant-IT PicoGreen dsDNA assay kit (Thermo Fisher) according to manufacturer's protocol. DNA was sheared to appropriate size with Covaris, followed by adaptor ligation. Sequencing was performed at New York University School of Medicine's Genome Technology Center.

\section{Quantitative RT-PCR}

Cells were grown until appropriate $O D_{600}$ and aliquots were collected and treated with RNAprotect Bacteria Reagent (Qiagen). After 5 minutes, cells were harvested and resuspended in lysis buffer (RNase-free TE buffer, $10 \mathrm{mg} \mathrm{ml}^{-1}$ lysozyme, $100 \mathrm{ug} \mathrm{ml}^{-1}$ ). Trizol LS (Thermo Scientific) was used according to manufacturer's protocol to extract total RNA. Samples were treated with DNase (Invitrogen) and purified using spin columns (Zymo Research). Superscript III reverse transcriptase (Invitrogen) was used to synthesize cDNA. qPCR reactions were amplified using Power SYBr Green PCR Master Mix (Applied Biosystems) with appropriate primer sets and cDNA template. 


\section{ChIP-seq}

ChIP was carried out as previously described with the following modifications ${ }^{30}$. Briefly, cells were grown at $37^{\circ} \mathrm{C}$ to $\mathrm{OD}_{600} \sim 0.4$ and a final concentration of $1 \%$ formaldehyde was added for in vivo cross-linking of nucleoprotein. A final concentration of $0.5 \mathrm{M}$ glycine was added to the culture to quench the reaction after a 20 minute incubation. Cells were collected by centrifugation and washing twice with 1X cold Tris-buffered saline then frozen in liquid nitrogen and stored at -80 . Cells were resuspended in lysis buffer $(50 \mathrm{mM}$ Tris [pH 7.5], 100mM NaCl, 1mM EDTA, protease inhibitor [Roche], $10 \mathrm{mg} \mathrm{ml}^{-1}$ lysozyme). After incubation at $37^{\circ} \mathrm{C}$, IP buffer (50mM HEPES-KOH, $150 \mathrm{mM} \mathrm{NaCl}, 1 \mathrm{mM}$ EDTA, $1 \%$ Triton $X 100,0.1 \%$ sodium deoxycholate, $0.1 \%$ SDS, protease inhibitor [Roche]) was added at a 1:3 ratio. DNA was sheared using ultrasonicator Covaris M220 on a 10 seconds on and 10 seconds off cycle for a total of 50 cycles.

The supernatant was incubated with 3xFLAG antibody (Biolegend) and Dynabeads Protein G (Thermo Scientific) overnight at $4^{\circ} \mathrm{C}$. Samples were then washed twice with IP buffer, once with IP buffer+500mM NaCl, once with wash buffer (10mM Tris, $250 \mathrm{mM} \mathrm{LiCl}, 1 \mathrm{mM}$ EDTA, $0.5 \%$ NP-40, 0.5\% sodium deoxycholate), and a final wash with TE. Immunoprecipitated complexes were eluted in elution buffer (50mM Tris, $10 \mathrm{mM}$ EDTA, $1 \% \mathrm{SDS}$ ) at $65^{\circ} \mathrm{C}$ for 20 minutes. Samples were treated with RNAse $\mathrm{A}$ (Qiagen), at $42^{\circ} \mathrm{C}$ and then uncross-linked with elution buffer+pronase for 2 hours at $42^{\circ} \mathrm{C}$, followed by 6 hours at $65^{\circ} \mathrm{C}$. DNA was purified using ChIP Clean and Concentrate (Zymo Research). Prior to sequencing, DNA was checked on TapeStation 2200 for appropriate size (Agilent). ChIP experiments were repeated for a total of three replicates.

For sequencing, sample libraries were prepared by using the NEBNext ChIP-seq Library (Illumina), according to manufacturer's protocol. Samples were sequenced on NextSeq 500 (Illumina). Bowtie and MACS2 were used for aligning and peak-calling, respectively ${ }^{10}$.

\section{Electrophoretic mobility shift assay}

Protein purification. YcjW and S258N YcjW were cloned into plasmid pet28SUMO using the Gibson Assembly Mastermix kit, according to the manufacturer's protocol (NEB). Auto-induction media was used for protein production (152). Cells were harvested and resuspended in lysis buffer ( $1 \mathrm{M} \mathrm{NaCl}, 5 \mathrm{mM}$ Imidaziole, $5 \%$ Glycerol, protease inhibitor cocktail [Roche]) and sonicated. AKTA Start system was used for chromatography with HisTrapHP columns (GE Healthcare Life Sciences). Columns were 
washed in wash buffer (50 mM Tris-Cl [pH 8.0], $10 \mathrm{mM}$ Imidazole, 5\% Glycerol, $500 \mathrm{mM}$ $\mathrm{NaCl}$ ), followed by gradient elution with elution buffer (50 mM Tris- $\mathrm{Cl}$ [pH 8.0], $250 \mathrm{mM}$ Imidazole, 5\% Glycerol, $250 \mathrm{mM} \mathrm{NaCl}$ ). The SUMO tag was cleaved with SUMO protease in dialysis buffer (200 mM NaCl, $50 \mathrm{mM}$ Tris-Cl [pH 8.0], $5 \%$ Glycerol, $1 \mathrm{mM}$ DTT). Samples were applied to a HiTrap HeparinHP column (GE). Columns were washed with buffer (20mM Tris [pH 8.0]. $50 \mathrm{mM} \mathrm{NaCl}, 5 \%$ glycerol), and eluted in elution buffer (20 mM Tris [pH 8.0], $1.5 \mathrm{M} \mathrm{NaCl}, 5 \%$ glycerol). The sample was concentrated to $5 \mathrm{~mL}$ and injected onto a Superdex 200 column with GF buffer (20 mM Tris-Cl [pH 8.0], $50 \mathrm{mM} \mathrm{NaCl}, 1 \mathrm{mM}$ DTT).

$E M S A$. dsDNA probes containing the binding sequence were radiolabeled with gamma ${ }^{32} \mathrm{P}$ rATP using T4 polynucleotide kinase (NEB). Labeled probes were purified by passage through size exclusion columns (Bio-Rad). Binding reactions were done as previously described ${ }^{12}$. The gel was then exposed to a phosphor screen and visualized on Storm 820 Phosphorimager (GE Healthcare). Experiments with various disaccharides were done in a similar fashion, except purified protein was incubated with appropriate sugar for 20 minutes at room temperature before addition of radiolabeled probe. 
a

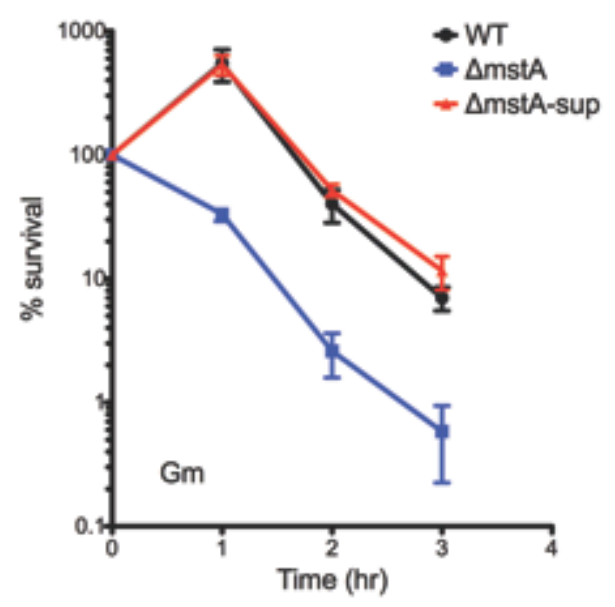

C

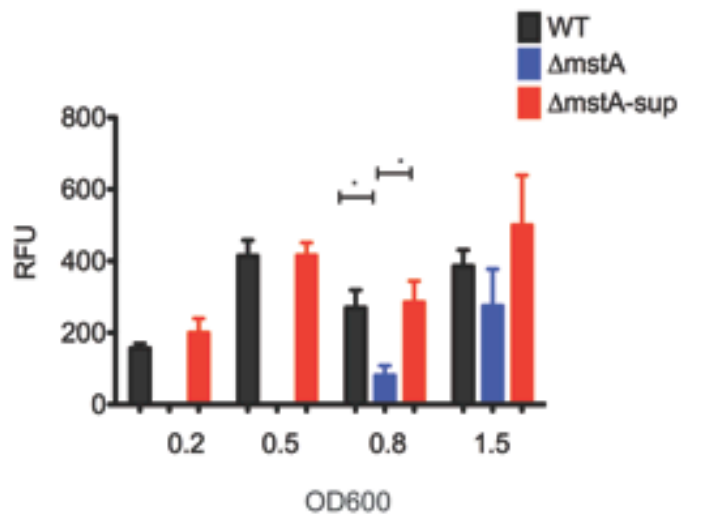

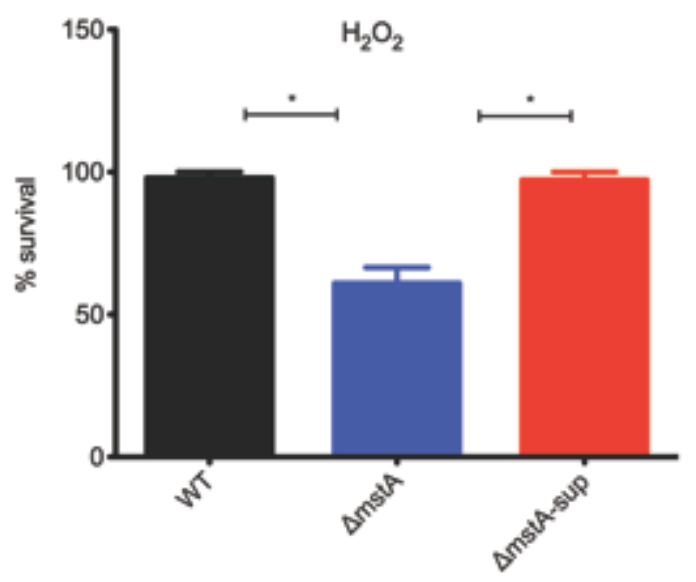

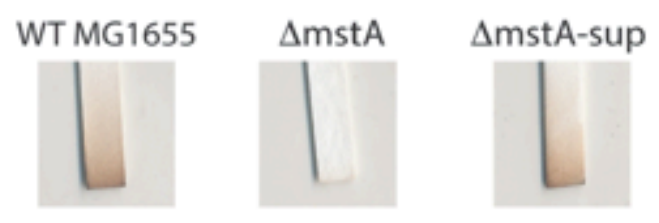

Figure 1 E. coli MG1655 lacking 3MSTA acquires phenotypic suppression and has increased $\mathrm{H}_{2} \mathrm{~S}$ levels and tolerance to $\mathrm{Gm}$ and $\mathrm{H}_{2} \mathrm{O}_{2}$ (a) $\triangle m s t A$-sup has increased survival rate compared to $\triangle m s t A$ when treated with $2 \mathrm{ug} \mathrm{m}^{-1} \mathrm{I}^{-}$gentamicin in a time-kill assay (b) $\triangle m s t A$-sup also has increased tolerance after exposure to $5 \mathrm{mM} \mathrm{H}_{2} \mathrm{O}_{2}$ for 30 minutes (c) $\mathrm{H}_{2} \mathrm{~S}$ production as measured with fluorescent probe, WSP5. Relative fluorescent units are normalized to $\mathrm{OD}_{600}$ and minus the background fluorescent of PBS buffer+100uM L-cysteine and WSP5. $\mathrm{H}_{2} \mathrm{~S}$ reacts with lead acetate, leading to staining of strips (Sigma-Aldrich). Values are means $\pm S D(n=3) .{ }^{*} p<0.05$ 
a
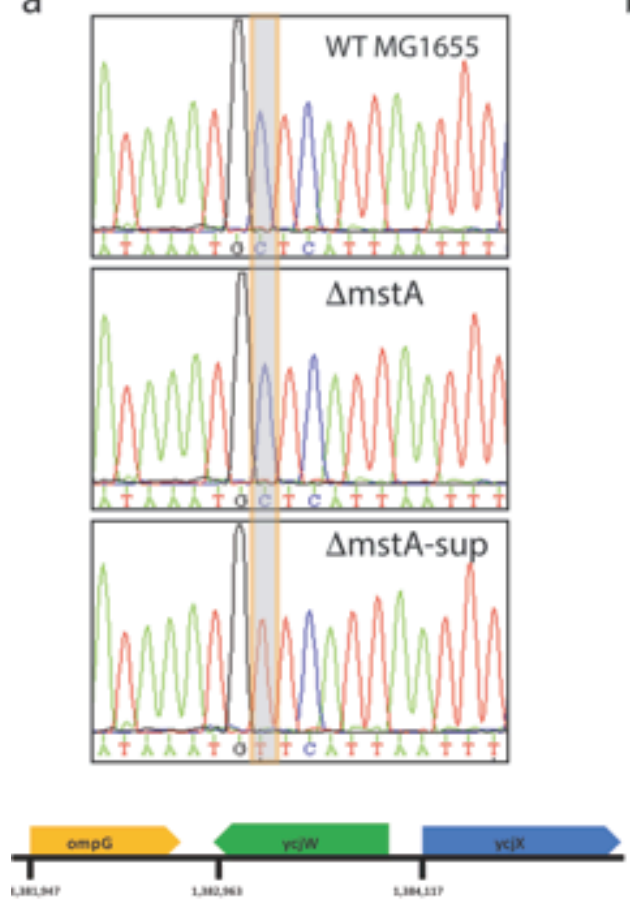

$\mathrm{C}$

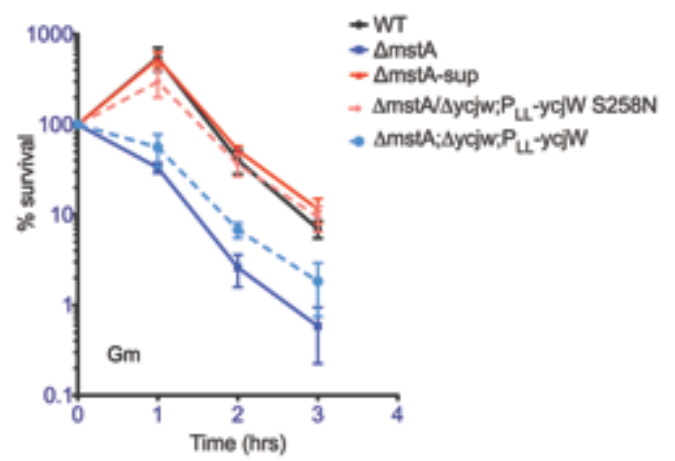

d b
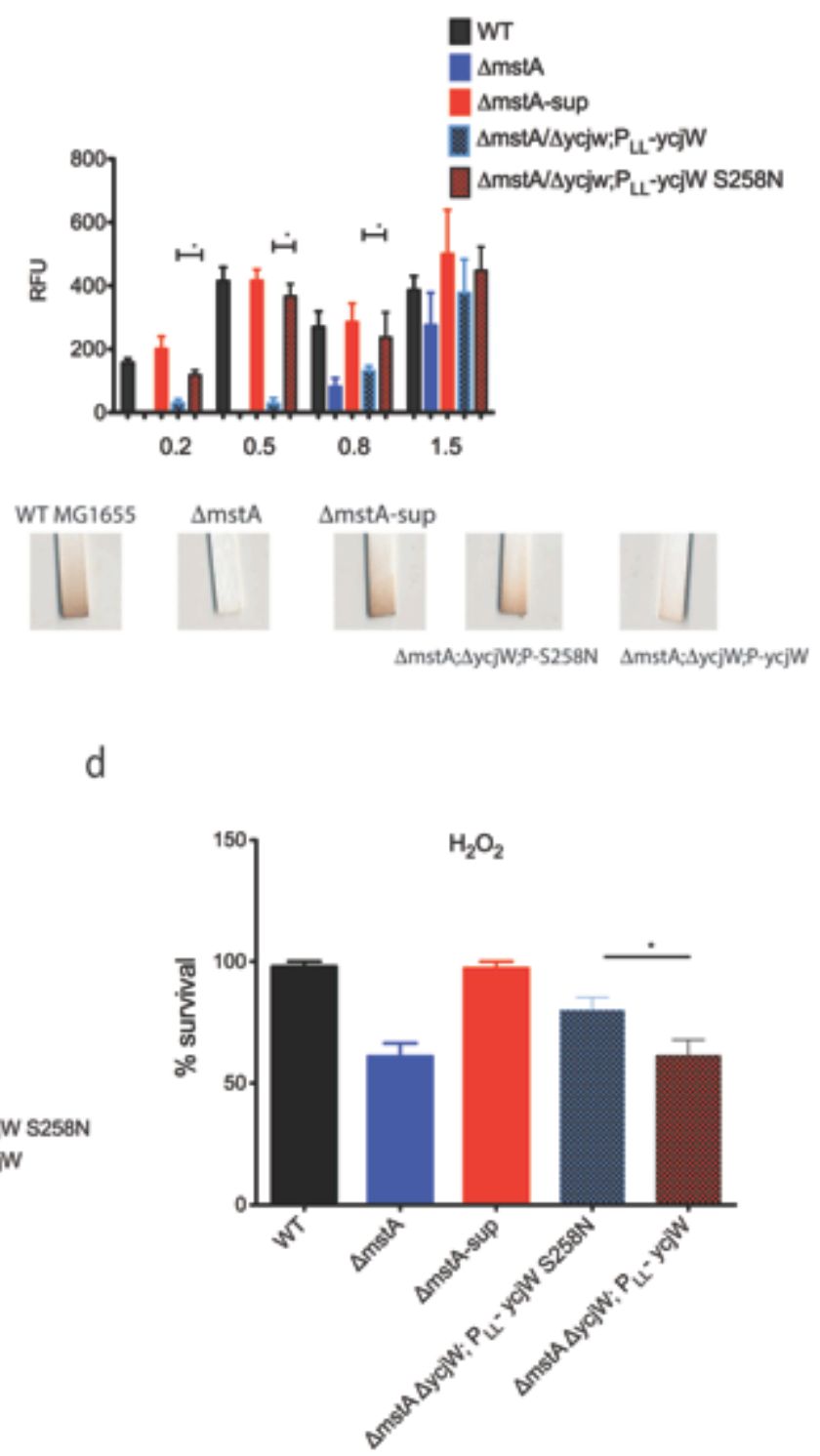

Figure 2 Unique nonsynonymous SNP in $\Delta m s t A$-sup is mapped to putative transcription factor $y c j W$. (a) PCR validation of whole genome sequencing. Displayed are sequences from $E$. coli MG155, $\Delta m s t A$, and $\Delta m s t A$-sup of (-) strand. The SNP changes amino acid 258 from serine to asparagine. (b) $\Delta m s t A I \Delta y c W$ strains with plasmid expressed $\mathrm{P}_{\mathrm{LL}-\mathrm{ycjW}}$ or $\mathrm{P}_{\mathrm{LL}-\mathrm{ycjW}} \mathrm{S} 258 \mathrm{~N}$ were measured for $\mathrm{H}_{2} \mathrm{~S}$ production. Only $\Delta m s t A I \Delta y c W ; \mathrm{P}_{\mathrm{LL}}-\mathrm{ycjW} \mathrm{S} 258 \mathrm{~N}$ had increased $\mathrm{H}_{2} \mathrm{~S}$ production with no significant differences with WT or $\Delta m s t A$-sup. At $\mathrm{OD}_{600} 1.5$ no significant differences exist

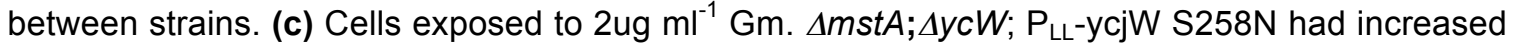

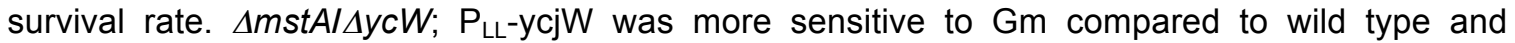
$\Delta m s t A$-sup. (d) $\Delta m s t A l \Delta y c W ; \mathrm{P}_{\mathrm{LL}}-\mathrm{ycjW} \mathrm{S} 258 \mathrm{~N}$ improved tolerance to $\mathrm{H}_{2} \mathrm{O}_{2}$. Values are means $\pm S D(n=3)$ for all experiments. 

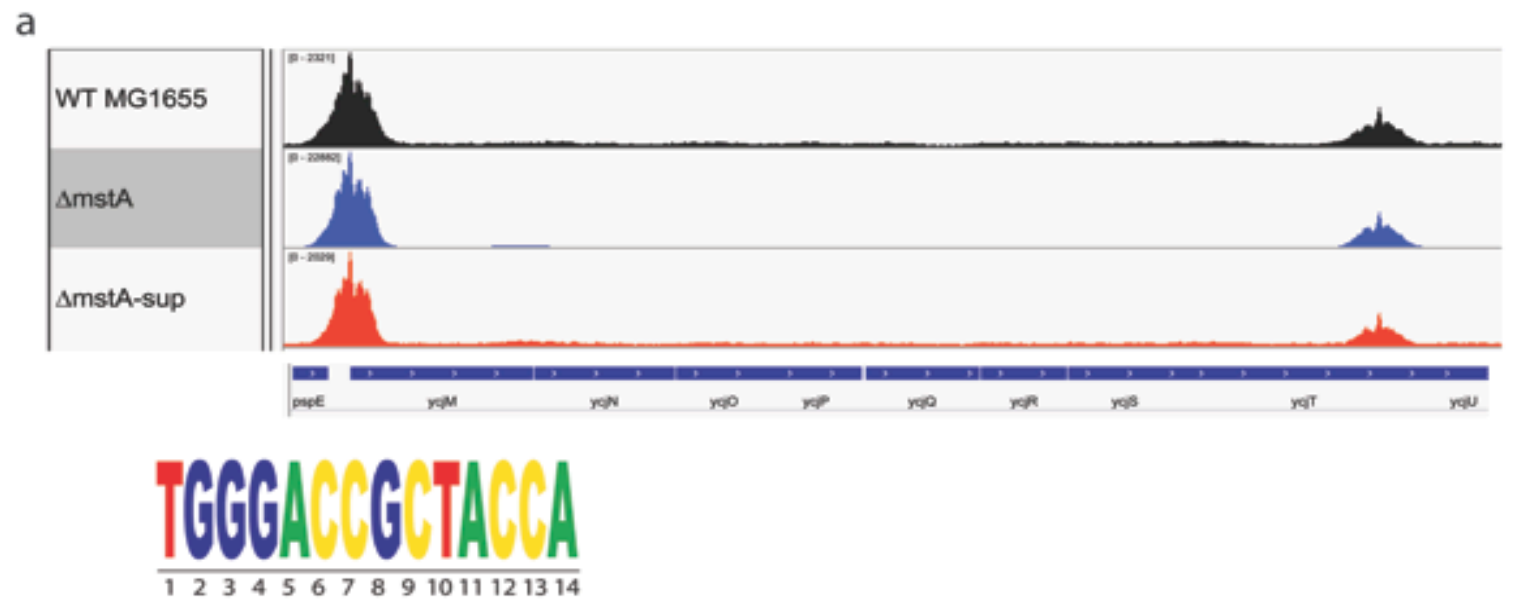

b

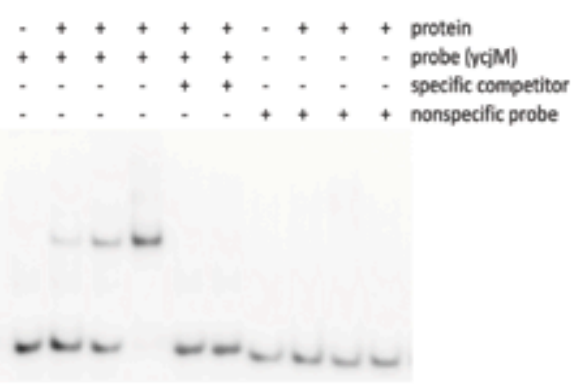

d
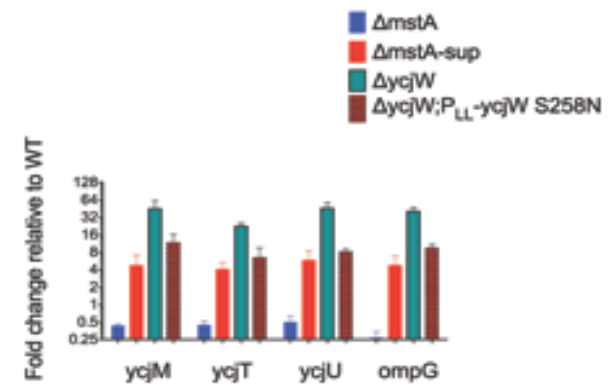

C
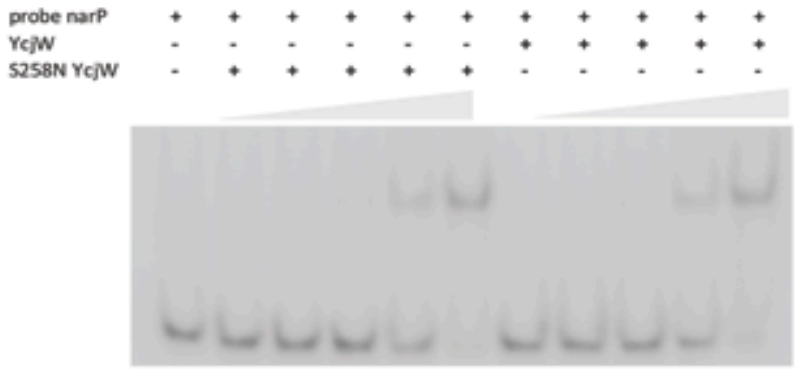

$\mathrm{e}$

Figure 3 YcjW, shows binding enrichment near ycjM and ycjU and regulates expression of operon ycjMNOPQRSTUV-ompG (a) Represented on Integrative Genomics Viewer (IGV), are sorted, aligned sequences containing pileup data to reference genome NC_000913.3. MACS ${ }^{10}$ was used for peak calling. Enriched peaks are upstream of ycjM and ycjU. A 14 nucleootide sequence identified as the binding motif for YcjW. (b) YcjW protein was titrated to DNA:protein ratios of 1:0.5, 1:1, and 1:2. Unlabeled ycjM probe was added to the reaction in excess to compete for binding. (c) YcjW and S258N YcjW protein were titrated to DNA:protein ratios of $1: 0.125,1: 0.25,1: 0.5,1: 1$, and 1:2. with narP probe. (d) qRT-PCR of a subset of genes in the $y c j M-V$ and ompG operon. The absence of $y c j W$ results in massive upregulation. $\triangle m s t A$-sup and $\triangle y c j W ; P_{L L}-y c j W$ both showed moderate and significant increased expression while mRNA levels are repressed in $\triangle m s t A$. Values are means \pm SD $(n=3)$. (e) YcjW protein was pre-incubated with Kojibiose, trehalose, or sucrose before radiolabeld DNA probes were added to the mixture. Only kojibiose prevented complex formation at a concentration of $0.5 \mathrm{mM}$. In contrast, both sucrose and trehalose added in excess at $5 \mathrm{mM}$ and $10 \mathrm{mM}$ failed to disrupt binding. 
a

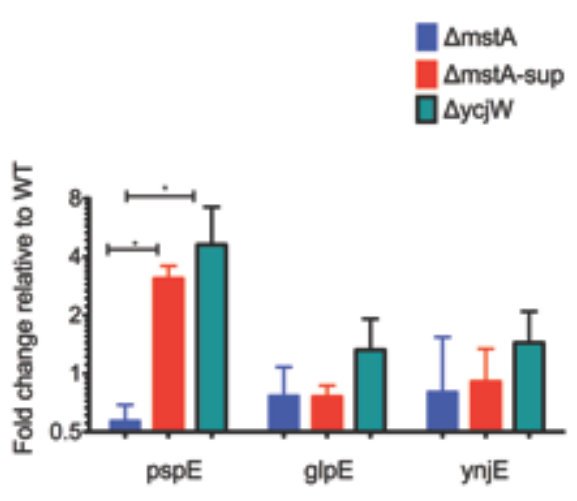

C

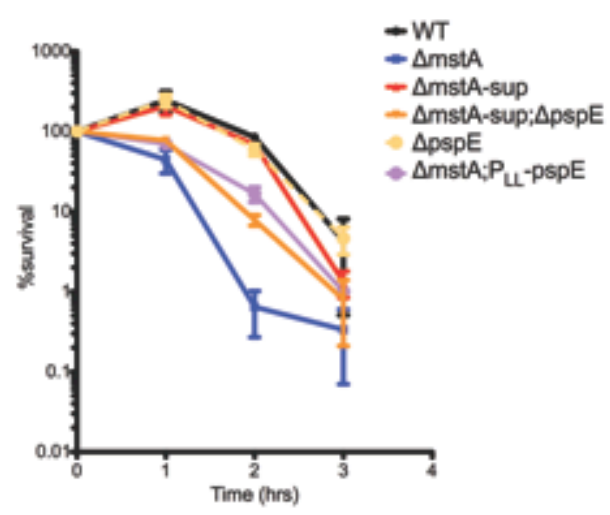

b

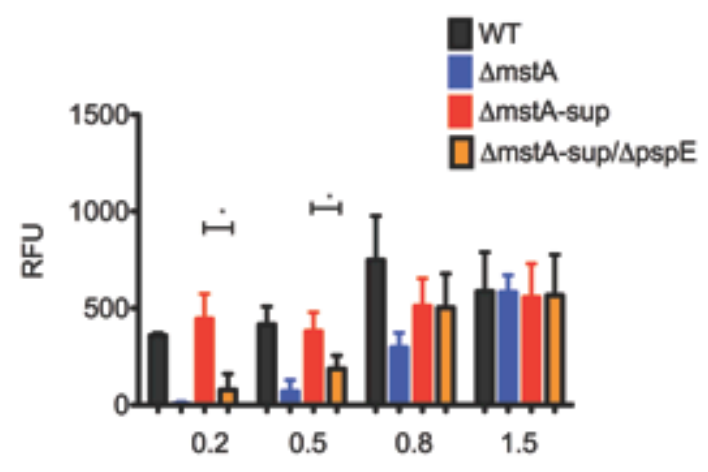

d

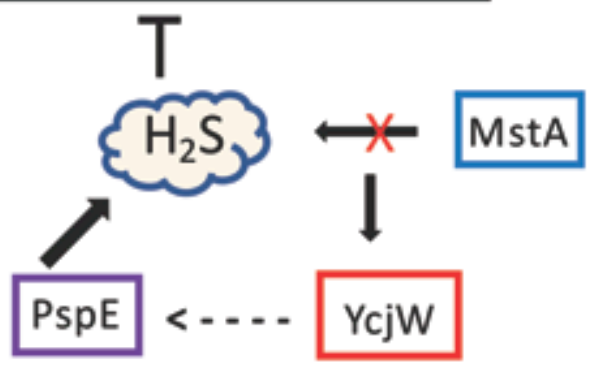

Figure 4 Deletion of pspE in $\Delta m s t A$-sup decreases $\mathrm{H}_{2} \mathrm{~S}$ biosynthesis during exponential growth (a)qRT-PCR of three thiosulfate sulfurtransferase genes. RNA was isolated from cells grown to $\mathrm{OD}_{600} \sim 0.4$. Only $p s p E$ expression was significantly increased in both $\Delta m s t A$-sup and $\Delta y c j W$ (b) $\mathrm{H}_{2} \mathrm{~S}$ levels in $\Delta m s t A$-sup; $\triangle p s p E$ were undetectable till $\mathrm{OD}_{600}$ reached 0.8. (c) $\Delta m s t A-$ sup/ $\Delta p s p E$ had increased sensitivity to gentamicin compared to WT and $\Delta m s t A$-sup. Overexpression of pspE in $\triangle m s t A$ increased tolerance to gentamicin comared to $\triangle m s t A$. Values are means $\pm S D(n=3)$ (d) In cells lacking MstA which is responsible for $\mathrm{H}_{2} \mathrm{~S}$ production, $\mathrm{S} 258 \mathrm{~N}$ YcjW upregulates PspE as an alternate route of $\mathrm{H}_{2} \mathrm{~S}$ biosynthesis. 


\section{TABLE 1}

\begin{tabular}{|c|c|c|}
\hline STRAIN NAME & Description & SOURCE \\
\hline E. coli K12 MG1655 & F- lambda- $i l v G-r f b-50 r p h-1$ & 3132 \\
\hline$\Delta m s t A$ & & 3 \\
\hline$\Delta m s t A$-sup & $\Delta m s t A ; y c j W^{\mathrm{s} 258 \mathrm{~N}}$ & This study \\
\hline$\Delta m s t A ; \Delta y c j W$ & & This study \\
\hline$\Delta m s t A ; \Delta y c j W ; \mathrm{P}_{\mathrm{LL}}-\mathrm{ycj} \mathrm{W}$ & & This study \\
\hline$\Delta m s t A ; \Delta y c j W ; \mathrm{P}_{\mathrm{LLy}} \mathrm{Cj} \mathrm{W}^{\mathrm{S} 258 \mathrm{~N}}$ & & This study \\
\hline$\Delta y c j W ; P_{\text {LLycjW }}$ S258N & & This study \\
\hline WT-3xFlag & $\begin{array}{l}\text { E. coli K12 MG1655 (ycjW fused to 3X } \\
\text { FLAG) }\end{array}$ & This study \\
\hline$\Delta m s t A$-3XFlag & $\Delta m s t A$ (ycjW fused to 3X FLAG) & This study \\
\hline$\Delta m s t A$-sup-3XFlag & $\begin{array}{l}\Delta m s t A ; y c j W^{\mathrm{s} 258 \mathrm{~N}}\left(y c j W^{\mathrm{s} 258 \mathrm{~N}} \text { fused to } 3 \mathrm{X}\right. \\
\text { FLAG) }\end{array}$ & This study \\
\hline$\Delta p s p E$ & & This study \\
\hline$\Delta m s t A$-sup; $\Delta p s p E$ & $\Delta m s t A ; y c j W^{\mathrm{s} 258 \mathrm{~N}} ; ; ; \Delta p s p E$ & This study \\
\hline$\Delta m s t A ; \mathrm{P}_{\mathrm{LL}}-\mathrm{pspE}$ & $\Delta m s t A$; overexpression PspE & This study \\
\hline
\end{tabular}


TABLE 2

\begin{tabular}{|c|c|c|}
\hline Name & Sequence & target \\
\hline LL01 & TTATACGCAAGGCGACAAGG & pKD3 $\mathrm{CmR}^{27}$ \\
\hline LL02 & GATCTTCCGTCACAGGTAGG & pKD3 $\mathrm{CmR}^{21}$ \\
\hline LL03 & CGGTGCCCTGAATGAACTGC & pKD4 KanR ${ }^{27}$ \\
\hline LL04 & CAGTCATAGCCGAATAGCCT & pKD4 KanR ${ }^{27}$ \\
\hline LL05 & CGGCCACAGTCGATGAATCC & pKD4 KanR ${ }^{27}$ \\
\hline LL06 & CACAATGGCCCGTTAGCAAC & mstA \\
\hline LL07 & TTTGCTGTGAAGCACCTGCG & mstA \\
\hline LL08 & AGTCGCTTCATTGCTGTCCT & ycjW up \\
\hline LL09 & CTTTCCGTTTCGCTGGAATA & ycjW down \\
\hline LL10 & gcataagggagagcgAGGCGCAGATGTCTGTCG & pAC-ycjW \\
\hline LL11 & gtagcccagcgcgtcTTATTTCAGCGTCATATGCG & pAC-ycjW \\
\hline LL12 & GAAATTAATGaacATTTATCGTGCGGCAGC & $\begin{array}{l}\text { pAC-ycjW } \\
\text { SN }\end{array}$ \\
\hline LL13 & AGGCTGTCAGTGGCAAAC & $\begin{array}{l}\text { pAC-ycjW } \\
\text { SN }\end{array}$ \\
\hline LL14 & CGGCGAACTCCATAAGATGT & 3xFlag ycjW \\
\hline LL15 & CTGGCATTCAGGCAAGGTAG & 3xFlag ycjW \\
\hline LL16 & CGACGTAAACTGTGCGGTAA & glpE \\
\hline LL17 & CGCCATGTAATCAACAAACG & glpE \\
\hline LL18 & GCA AAT GGG TTG AAG GAA AA & cysB \\
\hline LL19 & GCC GAA AAT AAC GCA AGA AA & cysB \\
\hline LL20 & TCGGGCTGCTAATAAACTGG & pspE \\
\hline LL21 & ATGTTGCCTTTCCGTGGTAG & pspE \\
\hline LL22 & $\begin{array}{l}\text { AATGGATGGCAATTTTTCATCCATAGAAGGAC } \\
\text { GCTTACATGGTGTAGGCTGGAGCTGCTTC }\end{array}$ & pspE-pKD3 \\
\hline LL23 & $\begin{array}{l}\text { ACCTTTGACCTTCGGCATTGCGATGTCTTTC } \\
\text { AGGCCACCGCATATGAATATCCTCCTTAG }\end{array}$ & pspE-pKD3 \\
\hline LL24 & GCCTTTAAAAAAGGCTTACTTGC & pspE-AskA \\
\hline LL25 & CCACCTTTGACCTTCGGCATTGC & pspE-AskA \\
\hline LL26 & CTAATGCCGGGTTACCTTGA & cyaA-qRT \\
\hline LL27 & GGCGGATCCTGTACTGACAT & cyaA-qRT \\
\hline LL28 & CGTGAACGTGAAATGTTTGG & narP-qRT \\
\hline LL29 & ATTCAACACCGAGGCAATCT & narP-qRT \\
\hline LL30 & CCGCAATAACGGTGAGTTTT & ompG-qRT \\
\hline LL31 & AAATCATGGCCTTCACGTTC & ompG-qRT \\
\hline LL44 & GATCAACTGGTTCACGCTCA & nrfA-qRT \\
\hline LL45 & CGTCCTGAATTGGCTTCATT & nrfA-qRT \\
\hline LL46 & TCGATGTTCGTGTTCCAGAG & pspE-qRT \\
\hline LL47 & ATCTCTTTTGCTTGCCCTGA & pspE-qRT \\
\hline LL48 & GCAGGCTTTCCATTTAACCA & glpE-qRT \\
\hline
\end{tabular}




\begin{tabular}{|c|c|c|}
\hline LL49 & GACCACATCGTAGCCCTGTT & glpE-qRT \\
\hline LL50 & ATCGTGAATGCCAACATCAA & pspA-qRT \\
\hline LL51 & TGTTCAATACGGCGAGTCAG & pspA-qRT \\
\hline LL52 & CGCGCTATTTCTGGCTATTC & pspB-qRT \\
\hline LL53 & TGCTCACTTTGCGACAATTC & pspB-qRT \\
\hline LL54 & AGCAGGCAAGCTGGTACTTC & pspC-qRT \\
\hline LL55 & CGGCGAGCAACTGATTTTAT & pspC-qRT \\
\hline LL56 & CGTGTTGTGAAATGTTGGGTTAA & $16 S$ \\
\hline LL57 & CCGCTGGCAACAAAGGATA & $16 \mathrm{~S}$ \\
\hline LL58 & $\begin{array}{l}\text { GAGTAGTCTCTCGTTTCATGGGACCGCTACC } \\
\text { ACGGAAAGGCAACatgAAAC }\end{array}$ & yсjM-EMSA \\
\hline LL59 & $\begin{array}{l}\text { GTTTCatGTTGCCTTTCCGTGGTAGCGGTCCC } \\
\text { ATGAAACGAGAGACTACTC }\end{array}$ & yсjM-EMSA \\
\hline LL60 & $\begin{array}{l}\text { CTAATGCCGGGTTACCTTGATGGTAACGTTC } \\
\text { CCAAAGGCATTTGCCTTTACACG }\end{array}$ & cyaA-EMSA \\
\hline LL61 & $\begin{array}{l}\text { CGTGTAAAGGCAAATGCCTTTGGGAACGTTA } \\
\text { CCATCAAGGTAACCCGGCATTAG }\end{array}$ & cyaA-EMSA \\
\hline LL62 & $\begin{array}{l}\text { GGTAACATTCACGCGCCTGGTAGCGTTACC } \\
\text { AACGCTACGCTCAAACATAATGATTC }\end{array}$ & narP-EMSA \\
\hline LL63 & $\begin{array}{l}\text { GAATCATTATGTTTGAGCGTAGCGTTGGTAA } \\
\text { CGCTACCAGGCGCGTGAATGTTACC }\end{array}$ & narP-EMSA \\
\hline LL64 & $\begin{array}{l}\text { CCTGCCAATCAGGATCACGATACGCCGCTA } \\
\text { TTACAGCAATATTTTTCGtga }\end{array}$ & nonspecific \\
\hline LL65 & $\begin{array}{l}\text { tcaCGAAAAATATTGCTGTAATAGCGGCGTAT } \\
\text { CGTGATCCTGATTGGCAGG }\end{array}$ & nonspecific \\
\hline LL66 & GTCGGCAAAAAGTGAATGGT & ycjM-qRT \\
\hline LL67 & GCATCTGGAATGGCGTTAAT & ycjM-qRt \\
\hline LL68 & CAACAGAAAACTGGCGTCAA & ycjT-qRT \\
\hline LL69 & CGCAGATGATAAAGCGCATA & ycjT-qRT \\
\hline LL70 & ACTGGCGTATCGCAAAATC & ycjU-qRT \\
\hline LL71 & GCATTCAGGGAGACAGAAGC & ycjU-qRT \\
\hline LL72 & CCGATGATCTGATGCTGGCT & rpoD-qRT \\
\hline LL73 & GTGCGCCCGATTTCAGATTC & rpoD-qRT \\
\hline
\end{tabular}




\section{SUPPLEMENTARY FIGURES}

$+2 \mathrm{ug} \mathrm{ml}^{-}$nalidixic acid

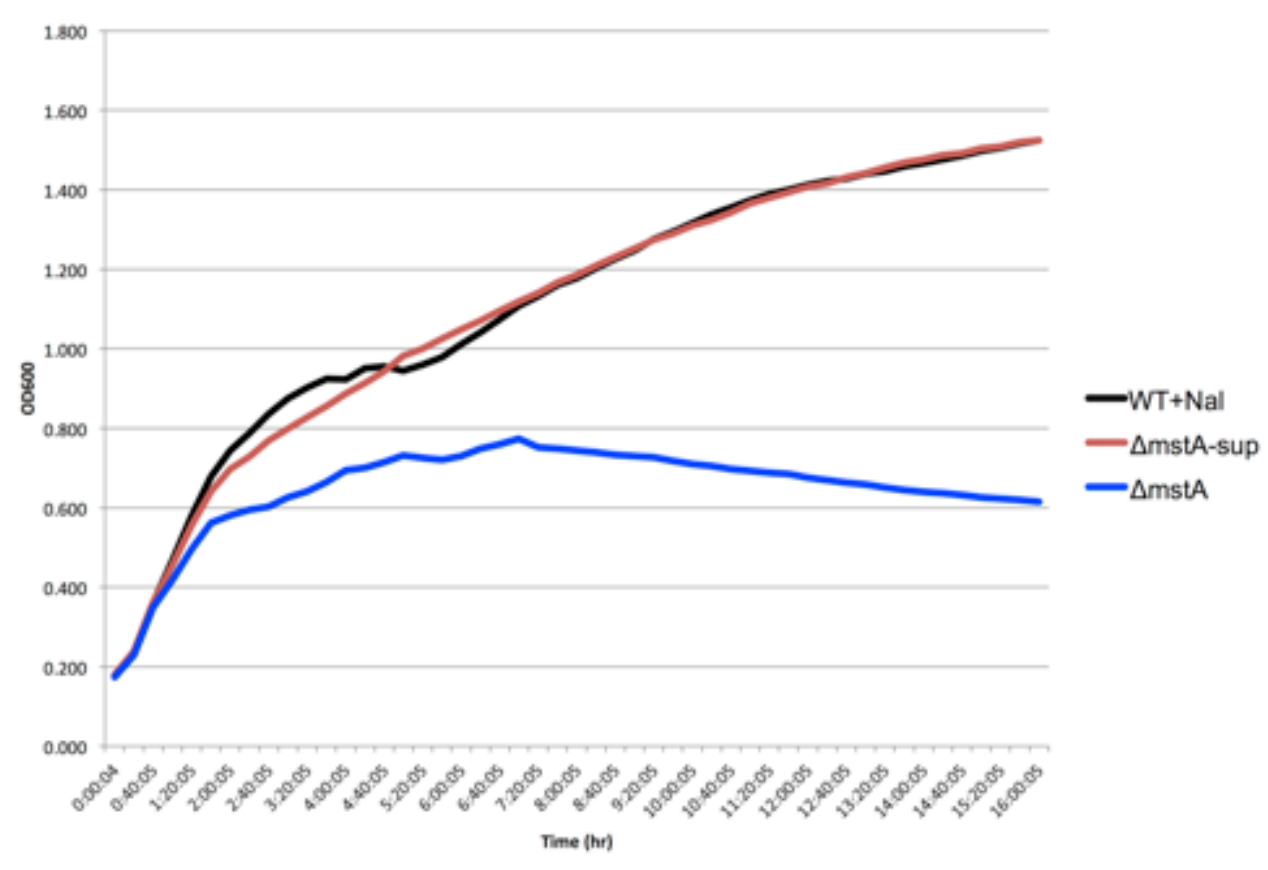

+1 ug ml- carbenicillin

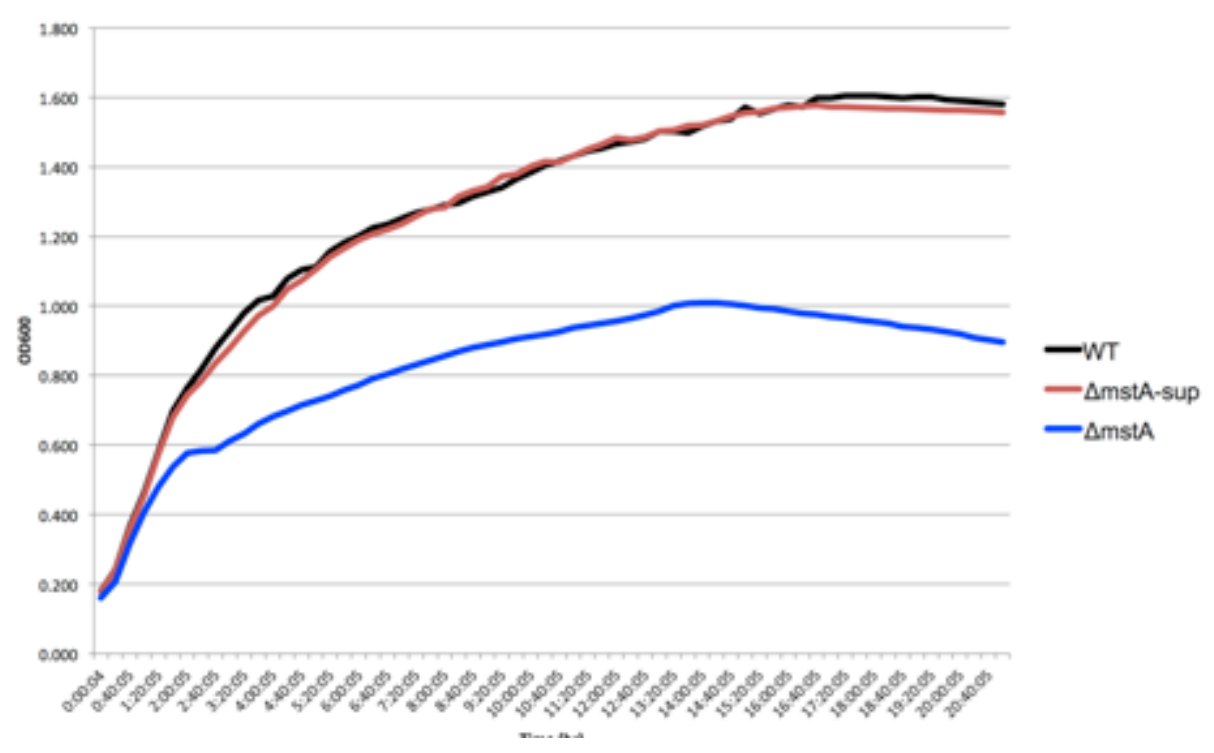

Fig. S1 $\Delta m s t A$-sup has increased tolerance to carbenicillin and nalidixic acid (a) Cells were grown in the presence of $2 \mathrm{ug} \mathrm{ml}^{-1}$ nalidixic acid and monitored for growth by $\mathrm{OD}_{600} . \Delta m s t A$-sup

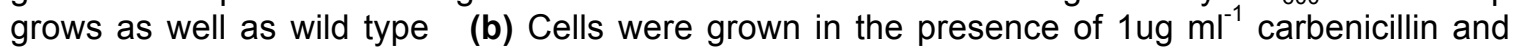
monitored for growth by $\mathrm{OD}_{600}$. $\Delta m s t A$-sup grows as well as wild type. 


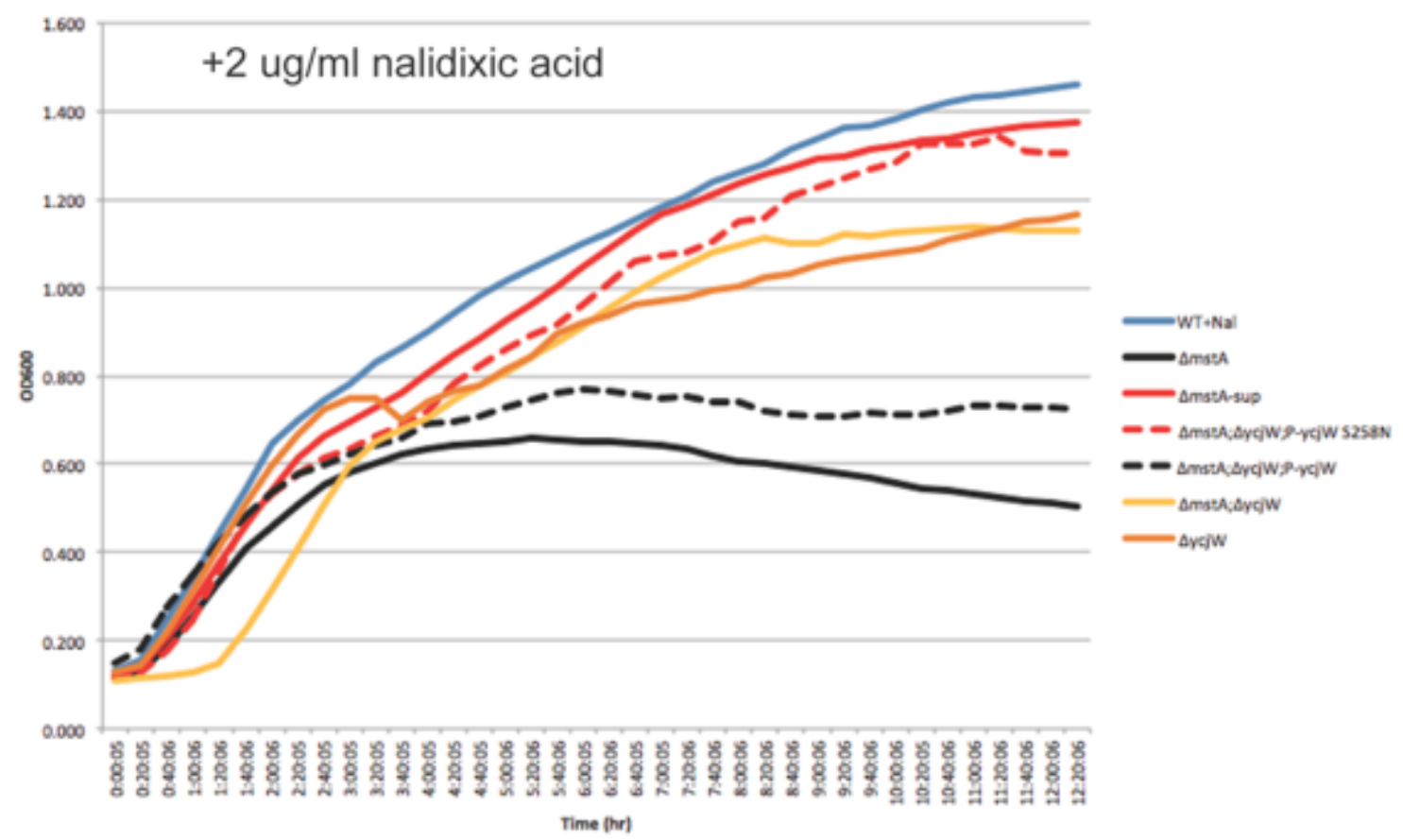

Fig. S2 Different strains of $\Delta y c j W$ and $\Delta m s t A$ mutant combinations, grown in the presence of nalidixic acid. $\Delta m s t A I \Delta y c W ; \mathrm{P}_{\mathrm{LL}-\mathrm{ycjW}} \mathrm{S} 258 \mathrm{~N}$ has similar growth rate to WT and $\Delta m s t A$-sup when grown in the presence of a sublethal concentration of nalidixic acid. In contrast, $\triangle m s t A l \Delta y c W ; \mathrm{P}_{\mathrm{LL}}-$ ycjW has a reduced growth rate compared to wild type and $\triangle m s t A$-sup. Interestingly, $\triangle y c j W$ also has impaired growth, though not to the extent of $\Delta m s t A$.

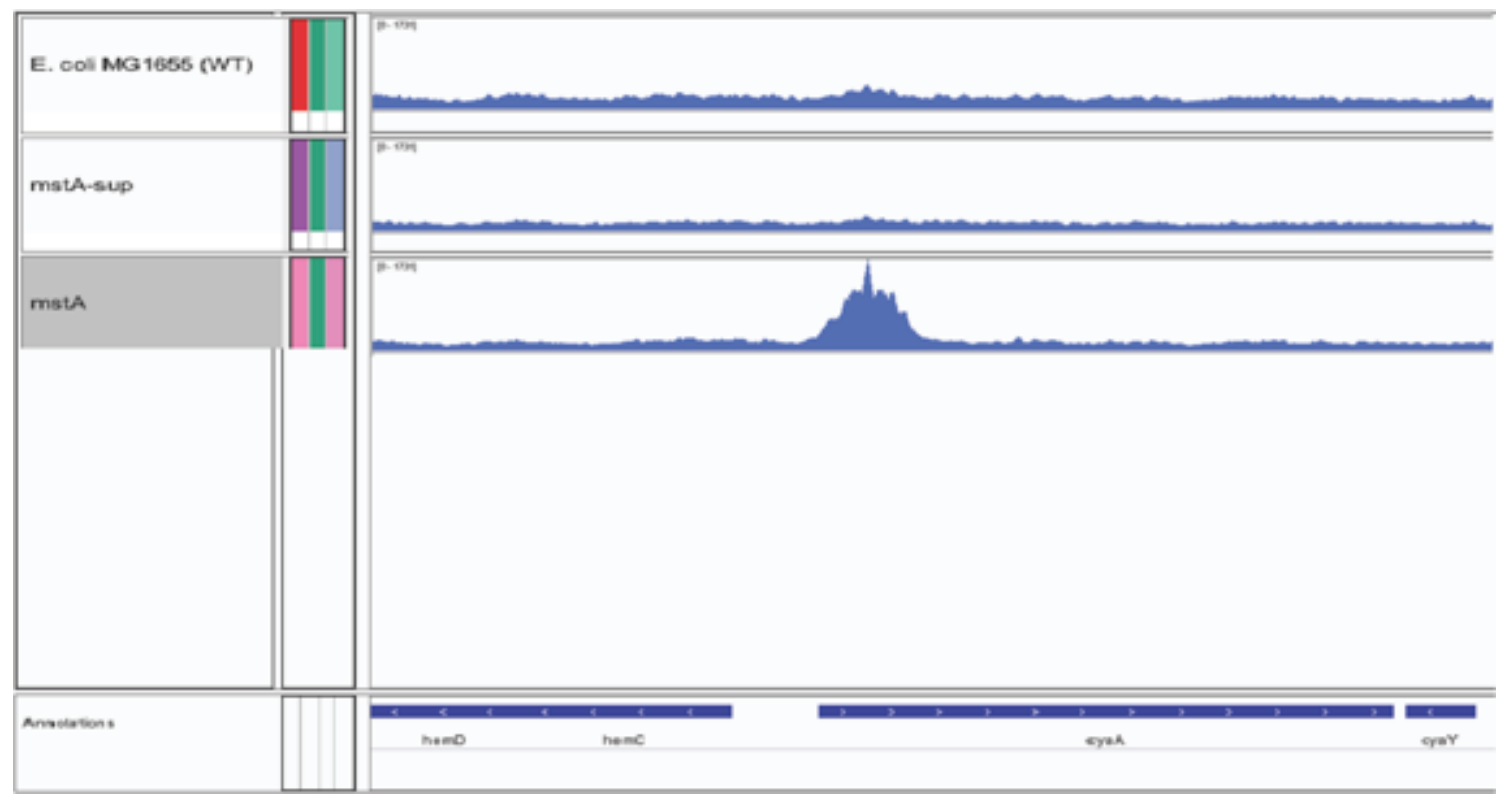

Fig. S3 ChIP-seq aligned data and pileup data visualized on IGV. Shown is the region near cyaA. Only $\Delta m s t A$ showed an enrichment of binding at a site downstream of the translation start site of cyaA 


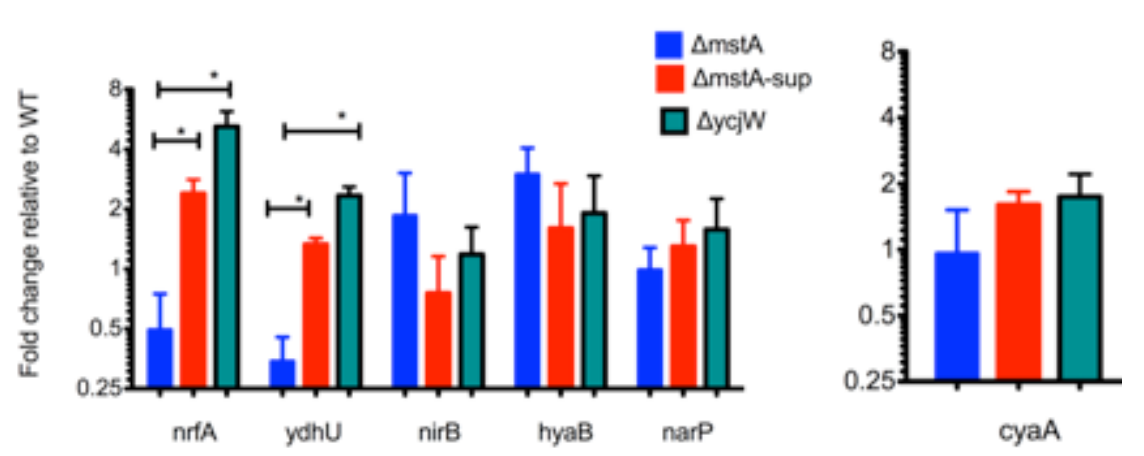

Fig. S4 YcjW does not affect narP expression or cyaA. NarP is a two-component nitrate/nitrite response regulator and can either repress or activate transcription. Of the genes measured for changes in mRNA levels, only nrfA and ydhU are significantly increased in $\triangle y c W$. Upregulation however, is modest.

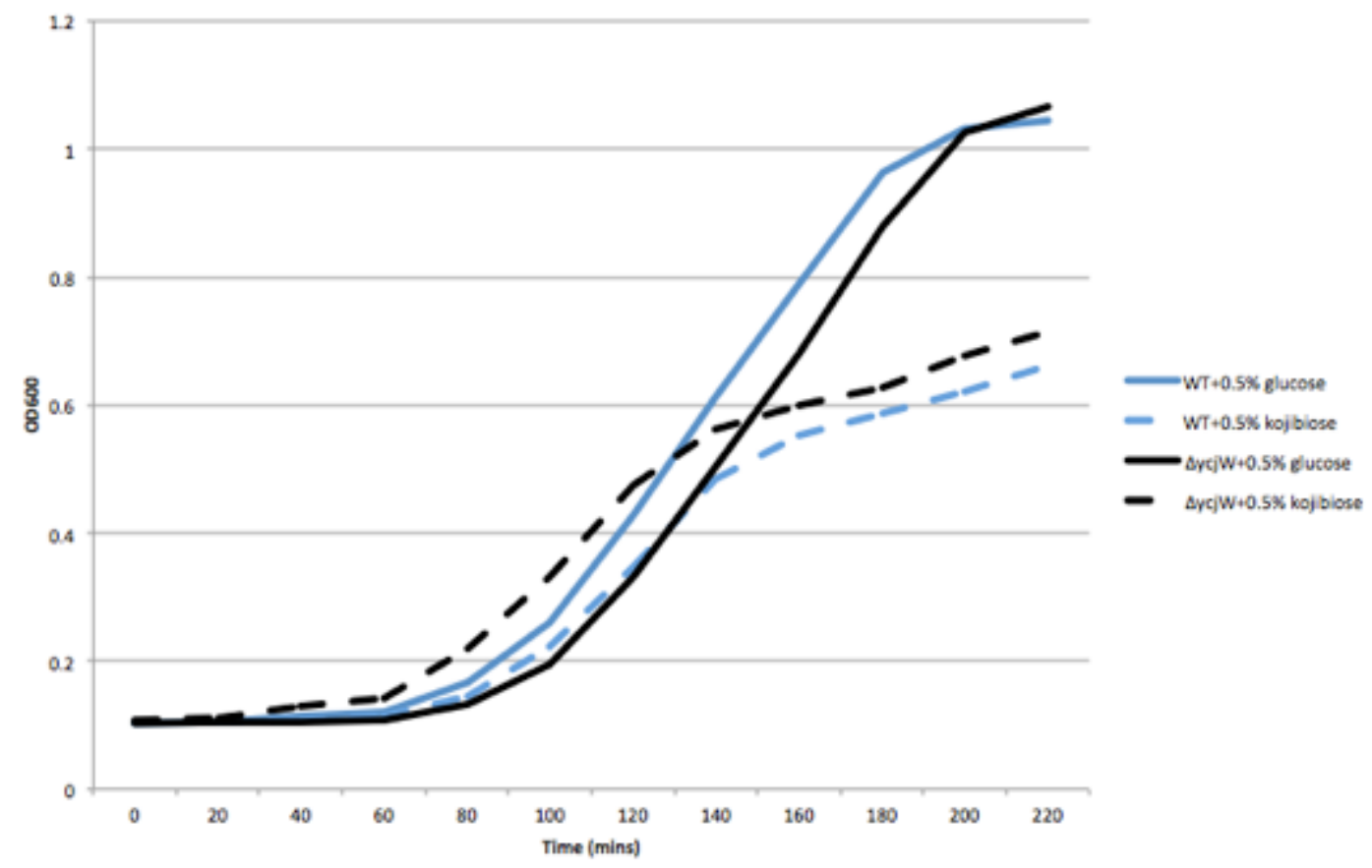

Fig S5 E. coli cells were grown in EZ rich defined media supplemented with either $0.5 \%$ glucose or $0.5 \%$ kojibiose as the carbon source. WT has a pronounced growth reduction with kojibiose. Knockout of $y c j W$, which results in significant upregulation of $y c j T$, capable of catalyzing kojibiose, did not improve growth. 


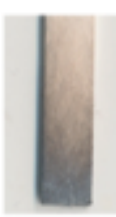

WT
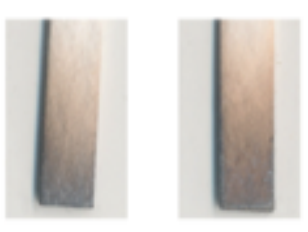

$\Delta$ mstA-sup $\Delta$ mstA-sup; $\Delta \mathrm{pspE}$
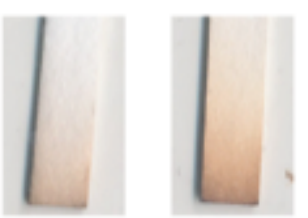

$\Delta m s t A \quad \Delta m s t A ; P-p s p E$

Fig S6 After overnight incubation with lead acetate strips, $\Delta m s t A$-sup/ $\Delta p s p E$ is able to overcome the early deficit in $\mathrm{H}_{2} \mathrm{~S}$ production seen during exponential growth. This is in contrast to $\triangle m s t A$, which does not accumulate extracellular $\mathrm{H}_{2} \mathrm{~S}$ to the extent of WTafter overnight growth.

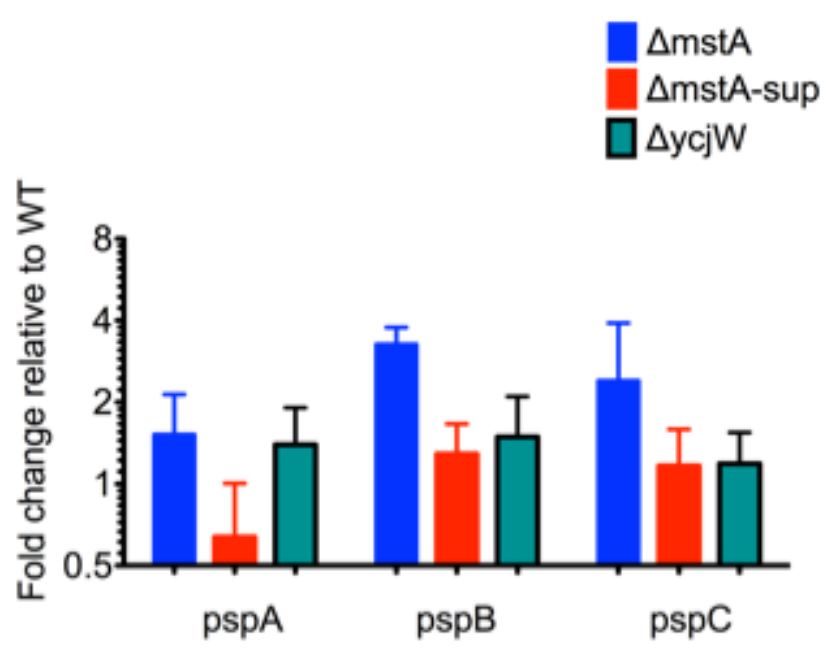

Fig $\mathbf{S} 7 \mathrm{YcjW}$ does not affect other $p s p$ genes. $p s p A B C$ is upstream of $p s p E$ which is upregulated in $\triangle y c W$ indicating that the regulation of $p s p E$ in this case is distinct and separate from the phage shock operon in general. 


\section{REFERENCES}

1 Kimura, H. Physiological roles of hydrogen sulfide and polysulfides. Nihon yakurigaku zasshi. Folia pharmacologica Japonica 147, 23-29, doi:10.1254/fpj.147.23 (2016).

2 Szabo, C. A timeline of hydrogen sulfide (H2S) research: From environmental toxin to biological mediator. Biochemical pharmacology 149, 5-19, doi:10.1016/j.bcp.2017.09.010 (2018).

3 Shatalin, K., Shatalina, E., Mironov, A. \& Nudler, E. H2S: a universal defense against antibiotics in bacteria. Science 334, 986-990, doi:10.1126/science.1209855 (2011).

4 Mironov, A. et al. Mechanism of H2S-mediated protection against oxidative stress in Escherichia coli. Proceedings of the National Academy of Sciences of the United States of America 114, 6022-6027, doi:10.1073/pnas.1703576114 (2017).

5 Shukla, P. et al. "On demand" redox buffering by H2S contributes to antibiotic resistance revealed by a bacteria-specific H2S donor. Chemical science $\mathbf{8}$, 4967-4972, doi:10.1039/c7sc00873b (2017).

6 Kolluru, G. K., Shen, X., Bir, S. C. \& Kevil, C. G. Hydrogen sulfide chemical biology: pathophysiological roles and detection. Nitric oxide : biology and chemistry 35, 5-20, doi:10.1016/j.niox.2013.07.002 (2013).

7 Luhachack, L. \& Nudler, E. Bacterial gasotransmitters: an innate defense against antibiotics. Current opinion in microbiology 21, 13-17, doi:10.1016/j.mib.2014.06.017 (2014).

8 Peng, B. et al. Fluorescent probes based on nucleophilic substitutioncyclization for hydrogen sulfide detection and bioimaging. Chemistry 20, 1010-1016, doi:10.1002/chem.201303757 (2014).

9 Fukami-Kobayashi, K., Tateno, Y. \& Nishikawa, K. Parallel evolution of ligand specificity between LacI/GalR family repressors and periplasmic sugarbinding proteins. Molecular biology and evolution 20, 267-277, doi:10.1093/molbev/msg038 (2003).

10 Feng, J., Liu, T., Qin, B., Zhang, Y. \& Liu, X. S. Identifying ChIP-seq enrichment using MACS. Nature protocols 7, 1728-1740, doi:10.1038/nprot.2012.101 (2012).

11 Ravcheev, D. A. et al. Comparative genomics and evolution of regulons of the LacI-family transcription factors. Frontiers in microbiology 5, 294, doi:10.3389/fmicb.2014.00294 (2014).

12 Zuo, Z. \& Stormo, G. D. High-resolution specificity from DNA sequencing highlights alternative modes of Lac repressor binding. Genetics 198, 13291343, doi:10.1534/genetics.114.170100 (2014).

13 Hall, B. G., Betts, P. W. \& Wootton, J. C. DNA sequence analysis of artificially evolved ebg enzyme and ebg repressor genes. Genetics 123, 635-648 (1989).

14 Lu, F., Brennan, R. G. \& Zalkin, H. Escherichia coli purine repressor: key residues for the allosteric transition between active and inactive conformations and for interdomain signaling. Biochemistry 37, 15680-15690, doi:10.1021/bi981617k (1998). 
15 Yamamoto, T. et al. Cloning and sequencing of kojibiose phosphorylase gene from Thermoanaerobacter brockii ATCC35047. Journal of bioscience and bioengineering 98, 99-106, doi:10.1016/S1389-1723(04)70249-2 (2004).

16 Jung, J. H., Seo, D. H., Holden, J. F. \& Park, C. S. Identification and characterization of an archaeal kojibiose catabolic pathway in the hyperthermophilic Pyrococcus sp. strain ST04. Journal of bacteriology 196, 1122-1131, doi:10.1128/JB.01222-13 (2014).

17 Kuznetsova, E. et al. Genome-wide analysis of substrate specificities of the Escherichia coli haloacid dehalogenase-like phosphatase family. The Journal of biological chemistry 281, 36149-36161, doi:10.1074/jbc.M605449200 (2006).

18 Mukherjee, K., Narindoshvili, T. \& Raushel, F. M. Discovery of a Kojibiose Phosphorylase in Escherichia coli K-12. Biochemistry 57, 2857-2867, doi:10.1021/acs.biochem.8b00392 (2018).

19 Franceus, J., Pinel, D. \& Desmet, T. Glucosylglycerate Phosphorylase, an Enzyme with Novel Specificity Involved in Compatible Solute Metabolism. Applied and environmental microbiology 83, doi:10.1128/AEM.01434-17 (2017).

20 Klahn, S., Steglich, C., Hess, W. R. \& Hagemann, M. Glucosylglycerate: a secondary compatible solute common to marine cyanobacteria from nitrogen-poor environments. Environmental microbiology 12, 83-94, doi:10.1111/j.1462-2920.2009.02045.x (2010).

21 Cheng, H., Donahue, J. L., Battle, S. E., Ray, W. K. \& Larson, T. J. Biochemical and Genetic Characterization of PspE and GlpE, Two Single-domain Sulfurtransferases of Escherichia coli. The open microbiology journal 2, 18-28, doi:10.2174/1874285800802010018 (2008).

22 Brissette, J. L., Weiner, L., Ripmaster, T. L. \& Model, P. Characterization and sequence of the Escherichia coli stress-induced psp operon. Journal of molecular biology 220, 35-48 (1991).

23 Jovanovic, G. et al. The N-terminal amphipathic helices determine regulatory and effector functions of phage shock protein A (PspA) in Escherichia coli. Journal of molecular biology 426, 1498-1511, doi:10.1016/j.jmb.2013.12.016 (2014).

24 Neidhardt, F. C., Bloch, P. L. \& Smith, D. F. Culture medium for enterobacteria. Journal of bacteriology 119, 736-747 (1974).

25 Baba, T. et al. Construction of Escherichia coli K-12 in-frame, single-gene knockout mutants: the Keio collection. Molecular systems biology 2, 2006 0008, doi:10.1038/msb4100050 (2006).

26 Thomason, L. C., Costantino, N. \& Court, D. L. E. coli genome manipulation by P1 transduction. Current protocols in molecular biology Chapter 1, Unit 1 17, doi:10.1002/0471142727.mb0117s79 (2007).

27 Datsenko, K. A. \& Wanner, B. L. One-step inactivation of chromosomal genes in Escherichia coli K-12 using PCR products. Proceedings of the National Academy of Sciences of the United States of America 97, 6640-6645, doi:10.1073/pnas.120163297 (2000). 
28 Seidman, C. E. \& Struhl, K. Introduction of plasmid DNA into cells. Current protocols in protein science Appendix 4, 4D, doi:10.1002/0471140864.psa04ds13 (2001).

29 Uzzau, S., Figueroa-Bossi, N., Rubino, S. \& Bossi, L. Epitope tagging of chromosomal genes in Salmonella. Proceedings of the National Academy of Sciences of the United States of America 98, 15264-15269, doi:10.1073/pnas.261348198 (2001).

30 Grainger, D. C. et al. Genomic studies with Escherichia coli MelR protein: applications of chromatin immunoprecipitation and microarrays. Journal of bacteriology 186, 6938-6943, doi:10.1128/JB.186.20.6938-6943.2004 (2004).

31 Bachmann, B., pp. 2460-2488.

32 Neidhardt, F. C. \& Curtiss, R. Escherichia coli and Salmonella : cellular and molecular biology. 2nd edn, (ASM Press, 1996). 